\title{
Begriffe des Bildungswesens und ihre Deutsch $\leftrightarrow$ Spanisch - Übersetzung
}

\author{
[Educational concepts and their German $\leftrightarrow$ Spanish translation] \\ http://dx.doi.org/10.11606/1982-8837254583
}

Dorit Heike Gruhn ${ }^{1}$

\begin{abstract}
The present study aims to outline German $\leftrightarrow$ Spanish equivalence of educational system concepts in the context of sworn translation of certificates. First of all, the translation of this text type is examined from the angles of different translation theories. Subsequently, the target audience and its specific needs are outlined from a functional perspective. Then examples of pairs of educational terms in the two languages are given, and they are organized in a three-part system (complete equivalence, partial equivalence and nonequivalence). Cases of "false friends" are identified as the most frequent source of errors. In addition, some translation tools and the most important strategies for the translation of this kind of concepts are presented. The frame of reference are the German and the Mexican educational systems.
\end{abstract}

Keywords: document translation; German $\leftrightarrow$ Spanish; educational systems.

Zusammenfassung: Der vorliegende Artikel gibt einen Überblick über die Deutsch $\leftrightarrow$ Spanisch - Äquivalenz von Begriffen des Bildungswesens in Bezug auf die Urkundenübersetzung. Zunächst wird die Übersetzung dieser Textsorte aus dem Blickwinkel verschiedener Übersetzungstheorien betrachtet. Nachfolgend werden - aus funktionaler Perspektive - die Adressaten der Zieltexte und ihre Bedürfnisse umrissen. Es folgen Beispiele für Fachausdrücke in beiden Sprachen, die in eine dreiteilige Systematik (vollständige Äquivalenz, Teiläquivalenz, Nicht-Äquivalenz) eingeordnet werden. Dabei stechen vor allem die "falschen Freunde" als häufige Fehlerquelle hervor. Eingegangen wird auch auf übersetzerische Hilfsmittel und die wichtigsten Übersetzungsstrategien im Rahmen der Übersetzung solcher Begriffe. Bezugssysteme sind das deutsche und das mexikanische Bildungswesen.

Stichwörter: Urkundenübersetzung; Deutsch $\leftrightarrow$ Spanisch; Bildungswesen.

\section{Die Übersetzung von Urkunden des Bildungswesens}

Neben Fachtexten aus dem Bereich Technik und Wirtschaft, gehören Dokumente mit öffentlich-rechtlichem Charakter zu den häufig übersetzten Textsorten². Letztere

\footnotetext{
${ }^{1}$ Benemérita Universidad Autónoma de Puebla, Facultad de Lenguas, 24 Norte 2003 Colonia Humboldt, Puebla, Puebla 72370, México. E-mail: heike50@hotmail.com. ORCID: 0000-0001-9024-5113

2 Genaue Zahlen sind mangels aktueller Studien nicht $\mathrm{zu}$ erhalten. Einschlägige Seiten von Übersetzungsagenturen, Übersetzerzusammenschlüssen u. ̈̈., deren Betreibern und Nutzern auf Grund ihrer berufsmäßigen Anbindung an diesen Bereich Glaubwürdigkeit zugesprochen werden kann, zählen
}

(cC) BY-NC

Pandaemonium, São Paulo, v. 25, n. 45, jan.-abr. 2022, p. 83-109 
GRUHN, D. H. - Deutsch $\leftrightarrow$ Spanisch - Übersetzuns

umfassen auch Urkunden des Bildungswesens ${ }^{3}$. In Zeiten des globalen Marktes, des Wissenstransfers und der exponentiellen Zunahme universitärer Austauschprogramme (wenngleich die Pandemie diese derzeit ausbremst) gehört die Einreichung von amtlich anerkannten Übersetzungen $\mathrm{zu}$ den unabdingbaren Bedingungen, um an Mobilitätsprozessen teilhaben zu können, sei es um in einem anderen Land zu studieren, $\mathrm{zu}$ forschen, $\mathrm{zu}$ arbeiten oder um firmenintern ins Ausland versetzt zu werden. „Die Übersetzung von Urkunden, also Bescheinigungen, Zeugnissen, Gerichtsurteilen [...] wird praktisch nur dann erforderlich, wenn damit ein Recht in einem anderen Land begründet werden soll“ heißt es bei Stolze (2013: 276).

Trotz ihres hohen Aufkommens wird Urkunden des Bildungswesens wenig Raum in übersetzungstheoretischen Schriften zugestanden. Das mag damit zu tun haben, dass praktizierende Übersetzer mehr Nutzen aus Handreichungen ziehen, als aus abstrakter Theorie $^{4}$. Aber auch mit der Schablonenhaftigkeit, die bei dieser Textsorte stärker ausgeprägt ist, als bei anderen Rechtstexten: Ihre Inhalte unterliegen gesetzlichen Vorgaben und Richtlinien von öffentlichen Stellen und Institutionen des Bildungswesens und sind repetitiv, die Sprache ist i.d.R. sachlich und trocken, der Satzbau fragmentarisch. Eine besondere translatorische Herausforderung scheint hier nicht zu liegen. Und dennoch: die Tücke steckt im Detail. Denn die Übersetzer ${ }^{5}$ solcher Dokumente, d.h. der Schul- und Universitätszeugnisse, Lehr- und Studienpläne, Prüfungsprotokolle, Abschlusstitel, Auszeichnungen, Diplome und Bescheinigungen, haben ein mitunter schwieriges Spagat zwischen unterschiedlichen nationalen Bildungssystemen zu leisten, d.h. zwischen Schul-, Ausbildungs- und Universitätswesen, curricularen und administrativen Strukturen, Titeln, Amtsbezeichnungen und Bildungsabschlüssen.

diese Dokumentensorte durchweg $\mathrm{zu}$ denen mit der höchsten Nachfrage (z.B.: https://uebersetzer.jetzt/info/uebersetzungspreise/uebersetzungspreise; Stand 15.01.2021). Bei einer von der Fundación Italia Morayta (2017: 50f.) in Mexiko unter knapp 1100 Übersetzern und Dolmetschern durchgeführten Umfrage erlangt das Themenfeld Recht den ersten Platz (mit 11,32\%) von insgesamt 29 Auswahloptionen (Einfachwahl). Die vorgenannten Angaben beziehen sich nicht auf bestimmte Sprachenpaare.

${ }^{3}$ Als Urkunden werden im rechtlichen Sprachgebrauch alle schriftlichen Dokumente mit Beweischarakter aufgefasst, also auch Nachweise des Bildungswesens (vgl. FLECK 1999: 231). Die vorliegende Arbeit bezieht sich in erster Linie auf solche Urkunden, die von einer amtlich bzw. institutionell befugten Autorität, in diesem Fall des Bildungswesens, ausgestellt worden sind, und somit Unterschrift und Stempel tragen. Vereinzelt sind auch Beispiele aus Lebensläufen einbezogen worden.

4 Ein umfassendes Werk für den deutschen Sprachraum diesbezüglich ist das Praxishandbuch Urkundenübersetzung. Fertigkeiten - Terminologie - Rechtssprache, von Radegundis Stolze (Stauffenburg Handbücher 2013).

${ }^{5}$ Personenbezeichnungen in der generischen maskulinen Form beziehen sich selbstverständlich auf beide Geschlechter. Die Verwendung dieser unterliegt Aspekten der Sprachökonomie, Stilistik und der besseren Lesbarkeit. 
GRUHN, D. H. - Deutsch $\leftrightarrow$ Spanisch - Übersetzuns

Innerhalb der von Alan Rey ausgearbeiteten Klassifizierung von Begriffssystemen, zit. nach Thiry (2008: 527), wären die Bildungssysteme den ,[...] sistemas nocionales prescriptivos o, por lo menos, legales, que ostentan una función organizadora y prescriptiva (códigos, discursos legales, institucionales, políticos, jurídicos $)^{\text {‘6 }}$ zuzurechnen, was laut Rey letztendlich die Unmöglichkeit beinhaltet, diese zu überstzen:

La consecuencia que se deriva de esta categorización es la imposibilidad de traducir, puesto que dichos sistemas constituyen una organización nocional relacionada con un discurso y, de ahí, con la existencia de una sociedad con una lengua natural particular y una organización institucional particular. (ebd.) ${ }^{7}$

Viele der in Bildungszusammenhängen verwendeten Begriffsbenennungen sind im Alltagsgebrauch verankert. Das verleitet $\mathrm{zu}$ vorschnellen Übertragungen in vermeintlich zielsprachige Entsprechungen, die der besonderen institutionellen Organisation von Ziel- und Ausgangsgesellschaft nicht gerecht werden. Vielmehr bleiben die in der ZS verwendeten Termini dem Horizont der Ausgangsgesellschaft verhaftet bzw. geben die einschlägigen Zusammenhänge der Ausgangsgesellschaft nicht sachgerecht wieder.

Ein Sachverständiger in Übersetzung (perito traductor) haftet für die Urkunden (die gestempelten Übersetzungen), die er ausgibt: Fehler oder Ungenauigkeiten können zur Annahme falscher Voraussetzungen führen und Rechte (des Übersetzungskunden) fälschlicherweise beschneiden oder gewähren.

Nachfolgend sollen ein summarischer Überblick über die Übersetzung von Begriffen aus dem Bildungswesen gegeben und anhand einer einfachen ÄquivalenzTypologie beispielhaft typische Schwierigkeiten für das Sprachenpaar Deutsch $\leftrightarrow$ Spanisch vorgestellt werden. Bezugspunkte sind dabei das deutsche und mexikanische Bildungssystem, da Begriffssysteme sich auch innerhalb von Nationalstaaten mit derselben Standardsprache (z.B. Mexiko und Argentinien oder Deutschland und Österreich) erheblich voneinander unterscheiden können. Ziel dieser Arbeit ist nicht die Kritik oder Weiterführung einer Theorie, unsere Überlegungen gründen auf der Praxis

\footnotetext{
${ }^{6}$ Übers. H.G.: [...] präskriptiven oder zumindest rechtlichen Begriffssystemen, die eine organisatorische und präskriptive Funktion aufweisen (Gesetzbücher, rechtliche, institutionelle, politische, juristische Diskurse).

${ }^{7}$ Übers. H.G.: Aus dieser Kategorisierung ergibt sich die Unmöglichkeit zu übersetzen, da die besagten Systeme eine Begriffsorganisation bilden, die mit einem Diskurs verbunden sind, und insofern mit der Existenz einer Gesellschaft, die eine besondere natürliche Sprache und eine besondere institutionelle Organisation aufweist.
} 
GRUHN, D. H. - Deutsch $\leftrightarrow$ Spanisch - Übersetzuns

und stellen den Versuch dar, praktische Erfahrungen zu beschreiben und einzuordnen. Sie wendet sich an alle Leser, die an dem Thema der Urkundenübersetzung Deutsch $\leftrightarrow$ Spanisch interessiert sind bzw. sich in dieses einarbeiten müssen ${ }^{8}$. Eine Beschreibung der jeweiligen Bildungssysteme ist nicht beabsichtigt. Bei den ausgewählten Beispielen handelt es sich ausschließlich um Nomen (nominale Simplizia und Komposita).

\section{Kurzübersicht: Ernennung zum „perito traductor" in Mexiko}

In Mexiko gibt es keine Möglichkeit, sich zum Übersetzer Deutsch $\leftrightarrow$ Spanisch ausbilden zu lassen. Die wenigen Übersetzungsstudiengänge, die angeboten werden ${ }^{9}$, konzentrieren sich auf die englische Sprache und bieten Deutsch bestenfalls als Dritt- oder Viertsprache an, wobei i.d.R. nur das Referenzrahmenniveau A2 oder B1 erreicht wird.

Eine Nachfrage nach Deutsch $\leftrightarrow$ Spanisch - Urkunden-Übersetzungen existiert in Mexiko vor allem in Ballungszentren, in denen deutsche Firmen und/oder große Universitäten ansässig sind (z.B. Mexiko-Stadt, Puebla, Querétaro).

Um behördlich anerkannte Übersetzungen anfertigen zu können, wird eine Ernennung als „perito traductor“ verlangt. Ein ,perito“ ist ein Sachverständiger, der von einer juristischen Instanz als solcher ernannt und ausgewiesen ist. „Peritos“ gibt es für viele Fachbereiche (z.B. für Industrieunfälle, Aktuarwesen u.v.m.). Nur in Ausnahmefällen ist das mit der Leistung eines Eids verbunden, die Bezeichnung „beeidigter Übersetzer“ ist also m.E. nicht zutreffend. Es gibt Aussschreibungen auf Bundesebene (Consejo de la Judicatura Federal - oberste juristische Verwaltungs- und Aufsichtsinstanz), teilweise auch über den Obersten Gerichtshof einzelner Bundesstaaten. Eine Schulung ist mit diesen Ausschreibungen nicht verbunden, für die Teilnahme sind anderweitig erworbene Qualifikationsnachweise einzureichen. Angesichts dessen, dass es keine einschlägige Ausbildung im Land gibt, muss davon ausgegangen werden, dass die meisten ,„peritos“ für Deutsch nicht über ein Übersetzerstudium verfügen.

\footnotetext{
${ }^{8}$ Was die angeführten Beispiele betrifft, so überwiegt die Übersetzungsrichtung Spanisch - Deutsch aufgrund der höheren Nachfrage in diese Richtung im Rahmen der Übersetzertätigkeit der Autorin.

${ }^{9}$ Grundständige Studiengänge (Licenciatura) in Übersetzung (Englisch): Universidad Nacional Autónoma de México (UNAM); Universidad Interamericana; Instituto Superior de Interpretación y Traducción (ISIT); Universidad Autónoma de Baja California (UABC). Einige dieser Institutionen bieten auch Spezialkurse oder 1-2-jährige Aufbaustudiengänge in Übersetzung an. El Colegio de México bietet einen Aufbaustudiengang in literarischer Übersetzung, der bis vor wenigen Jahren auch Deutsch führte.
} 


\section{Die Übersetzung von Urkunden des Bildungswesens im}

\section{Licht verschiedener Übersetzungstheorien}

Die Übersetzung von Urkunden des Bildungswesens findet zwar, wie oben schon angedeutet, selten explizit Beachtung in der Übersetzungswissenschaft, doch lässt sie sich umstandslos in gängige Theorien verschiedener Autoren einordnen.

Von Friedrich Schleiermacher (1813) über Antoine Berman (1985) bis hin zu Lawrence Venuti (1995) zieht sich die übersetzungstheoretische Frage, ob ein Translat sich so nah wie möglich am Ausgangstext zu bewegen habe und somit dem Adressaten ein gewisses $\mathrm{Maß}$ an Fremdheit zustehen sollte, oder so weit wie möglich an die (sprachlichen und kulturellen) Normen der Zielgesellschaft heranzutragen sei. Diese Dichotomie ist gegenwärtig nach Venuti als "Verfremdung" und "Domestizierung" bekannt und alle drei Autoren geben unter unterschiedlichen Gesichtspunkten der ersteren den Vorzug. Entstanden sind ihre Theorien im Rahmen der Literaturübersetzung, was aber nicht ausschließt, auch bezüglich der Urkundenübersetzung darüber nachzudenken. Diese muss eng an AT und Sachverhalte der fremden Ausgangsgesellschaft angelehnt bleiben:

[...] En effet, à la différence, par exemple, de la traduction litéraire ou de la traduction scientifique et technique, le lecteur du texte d'arrivée doit se rendre compte à tout moment qu'il s'agit d'une traduction. En aucun cas il ne saurait s'agir de "naturaliser" le plus possible le texte d'arrivé. (HENNEQUIN 2008: 77) ${ }^{10}$

Fachbegriffe dürfen also nicht „eingebürgert“" werden, um den Adressaten den Eindruck zu vermitteln, sie hätten es mit ,heimischen“ Strukturen des Bildungswesens zu tun. Ein Seminar zur „Mexikanischen Revolution“ ist also keinesfalls in „Deutsche Revolution von 1918/19“ umzutaufen, auch wenn beide demselben Jahrzehnt entstammen.

Die Frage, wo die Urkundenübersetzung im Rahmen der von Juliane House (2005) entwickelten Kategorien steht, wird im vorgenannten Zitat bereits mitbeantwortet. Es handelt sich zweifelsohne um eine offene Übersetzung, die Namen, Unterschrift und Stempel des Übersetzers trägt und deshalb jederzeit als solche erkennbar ist. Das ist

\footnotetext{
${ }^{10}$ Übers. H.G.: Im Gegensatz zu dem, was auf die literarische Überstetzung oder die wissenschaftlichtechnische Übersetzung zutrifft, muss dem Leser des Zieltextes hier in jedem Moment bewusst sein, dass es sich um eine Übersetzung handelt. Es kann mitnichten darum gehen, den Zieltext so weit wie möglich „einzubürgern“.
} 
GRUHN, D. H. - Deutsch $\leftrightarrow$ Spanisch - Übersetzung

insofern von Bedeutung, als es dem Übersetzer ausdrücklich erlaubt, erklärende Einfügungen und Fußnoten anzubringen (im Gegensatz zur verdeckten Übersetzung, die den Anschein erwecken soll, selbst ein Original zu sein).

Nach der Skopos-Theorie von Reiß und Vermeer (1984) ist „der Zweck (Skopos) die ,Dominante aller Translation“ (zit. nach SIEVER 2015: 85), wobei „die Funktion des Ausgangstexts nicht von vorneherein mit der des Zieltexts indentisch sein muss“ (ebd.: 87). In der Tat haben Übersetzungen von Urkunden des Bildungswesens im Vergleich zu den Originaldokumenten einen anderen bzw. erweiterten Zweck: Es geht nicht mehr nur darum, z.B. den Abschluss einer bestimmten Bildungsstufe innerhalb des eigenen nationalen Systems nachweisen zu können, ihr Skopos ist nun ein bürokratischer Akt in einem anderen nationalen Bildungssystem.

Eine Weiterentwicklung der Skopos-Theorie (vor allem mit der Absicht, diesen didaktisch in der Übersetzerausbildung umzusetzen) bildet der Ansatz von Christiane Nord, die Übersetzen primär als kommunikatives Handeln wertet. Sie führt die Begriffe „Funktionsgerechtigkeit“ und „Loyalität“ in die übersetzungstheoretische Diskussion ein, was im Vergleich zu Reiß/Vermeer wieder eine stärkere Betonung der Anbindung an den Ausgangstext (AT) beinhaltet, und sowohl bezüglich dessen Autor(en), als auch der Zielleser Loyalität einfordert. Nord unterscheidet zwei Übersetzungstypen, nämlich die „'dokumentarische Übersetzung' (Typ A) und 'instrumentelle Übersetzung' (Typ B) [...]“ (NORD 1999: 142f.). Während die dokumentarischen Übersetzungsformen Kommunikationssituation in der Ausgangskultur ${ }^{11}$ für die Zielleser dokumentieren (und somit offene Übersetzungen sind) können instrumentelle die gleiche Funktion haben wie nicht übersetzte Texte (vgl. ebd.). Die Übersetzung von Urkunden des Bildungswesens erfüllt die Kriterien der dokumentarischen Übersetzung. Allerdings mit der Einschränkung, dass ihr übergeordneter Zweck instrumenteller Natur ist, denn mit den ZT soll in der Zielgesellschaft gehandelt werden.

Wir sind geneigt, als Fazit dieses Kapitels für die Urkundenübersetzung ihre enge dokumentarische Anbindung an den AT als Leitlinie festzuhalten, des Weiteren den Skopos, das heißt in diesem Fall den Funktionswandel des anzufertigenden Translats im Vergleich zum AT. Und dem Praktiker darüber hinaus die Devise von Hans Hönig (1995:

\footnotetext{
${ }^{11}$ In diesem Text wird nicht von Ausgangs- bzw. Zielkultur, sondern von Ausgangs- und Zielgesellschaft bzw. -staaten gesprochen, da weder Nationalstaaten noch Sprachen homogene Kulturen zugeordnet werden können.
} 
GRUHN, D. H. - Deutsch $\leftrightarrow$ Spanisch - Übersetzuns

116, hier zit. nach SIEVER 2015: 96) nahezulegen: „Funktionierende Übersetzungen sind das Resultat intelligenten Verhaltens“.

\section{Die Adressaten der Übersetzung}

Wie bei jeder anderen Textsorte auch, ist aus funktionaler Sicht zunächst einmal das Profil der Adressaten solcher Urkundenübersetzungen zu umreißen. Wer wird die Übersetzungen lesen? Der Kunde meist nicht. Die eigentlichen Adressaten sind die Sachbearbeiter oder Beamten, die das Originaldokument anhand der Übersetzung einschätzen bzw. bewerten und auf dessen Grundlage amtliche Entscheidungen treffen müssen (Antragsannahme oder -ablehnung). Daraus ergibt sich, dass der Übersetzer den Zweck der Übersetzung (die Art des Antrags) kennen muss, um einschätzen zu können, welche Inhalte für diesen Zweck besonders relevant sind. Geht es zum Beispiel um die Anerkennung eines Studienabschlusses, so darf die Natur dieses Abschlusses (Fachbereich, Bildungsstufe, Ergebnis, erteilende Institution usw.) im ZT keine Zweifel aufkommen lassen. Andererseits ist dabei unerheblich, was genau die Amtsbezeichnung des Unterzeichners einer Apostille beinhaltet (z.B. Unterbereichsleiter). Erkennbar sein muss, dass die Apostille von einer dazu befugten Amtsperson unterzeichnet wurde. Oder ein deutsches Schulzeugnis von einer befugten Lehrperson. Denn für den Adressaten in Mexiko ist nicht relevant, was genau einen Studienrat oder Oberstudiendirektor auszeichnet. Es bietet sich eine Lehnübersetzung (wörtliche Übertragung bzw. Nachbildung von zusammengesetzten Wörtern der AS in der ZS) an (,director superior de estudios“" z.B.), auch wenn damit Benennungen geschaffen werden, die auf keinen Begriff in der ZS verweisen (also inhaltsleer sind), denn diese Amtskategorien existieren im mexikanischen Schulwesen nicht.

Typische Adressaten von Translaten ins Deutsche sind Konsulatrabteilungen (Visavergabe), Universitäten und Stellen für Studienbewerbung (z.B. uni-assist), Stipendienvergabe u.Ä., Vermittlerinstitutionen für bestimmte Berufsgruppen (z.B. die Firma Concura für Kranken- und Altenpfleger ${ }^{12}$ ), Arbeitgeber sowie Studienanerkennungsbehörden in Deutschland; von Translaten ins (mexikanische) Spanisch das Regierungssekretariat für Öffentliches Erziehungswesen (SEP), das über alle Gleichstellungs- und Anerkennungsanträge entscheidet, sowie Universitäten,

\footnotetext{
12 https://concura.mx/
} 
GRUHN, D. H. - Deutsch $\leftrightarrow$ Spanisch - Übersetzuns

Schulen oder Arbeitgeber. Nicht immer weiß der Kunde von vorneherein, an wie vielen Stellen (und in welchen Bundesstaaten) er die Übersetzungen einreichen wird. Manche Kunden schicken Studienbewerbungen an mehrere Universitäten in verschiedenen Bundesländern. Wenn beim Übersetzungsauftrag nicht ausdrücklich etwas anderes vorgegeben ist, muss davon ausgegangen werden, dass das Translat immer in erster Linie an behördliche Stellen (mit wechselndem Personal!) gehen, die Anträge verschiedener Fachrichtungen und Bildungseinrichtungen bearbeiten. Das bedeutet, dass es durchweg für Adressaten mitgedacht sein muss, die keine Spezialisten in dem jeweiligen Ausbildungs- oder Studienbereich und mit dem Bildungswesen der Ausgangsgesellschaft nicht vertraut sind. Insofern ist das Auftrags- und Adressatenprofil dieses Übersetzungstyps relativ homogen. Die im vorliegenden Text aufgeführten Beispiele sind allgemein gehalten und orientieren sich an diesem Profil.

Das „Übersetzerhandwerk“ wird von Richtlinien geleitet, die zumeist von Übersetzerverbänden stammen (BDÜ, $\mathrm{OMT}^{13}$ ) und letztendlich lediglich empfehlenden Charakter haben (vgl. FLECK 1999: 230). Weder in Deutschland noch in Mexiko gibt es diesbezüglich bislang rechtsverbindliche Vorschriften. Als Beispiel soll die folgende „Allgemeine Leitlinie für die Anfertigung von Urkundenübersetzungen in Bayern“ angeführt werden, die eng auf die Bedürfnisse der Übersetzungs-Adressaten zugeschnitten ist:

\section{Zeugnisse und Diplome}

5.1 Bei Zeugnissen und Diplomen sind der Schultyp, die Noten und die erworbene Berufsbezeichnung möglichst wörtlich zu übersetzen (Originalbezeichnung in Klammern oder als Fußnote wiederholen); das Auffinden einer inhaltlich entsprechenden Bezeichnung in der Zielsprache ist nicht Aufgabe des Übersetzers.

In einer Anmerkung kann eine inhaltlich entsprechende deutsche bzw. fremdsprachige Bezeichnung angegeben werden. Hierbei ist eine Formulierung, die als Präjudiz für ein Feststellungs- oder Genehmigungsverfahren missverstanden werden kann oder eine Verwendung von Rechtsbegriffen des hiesigen Schulwesens und die Verwendung von vorgegebenen Abschlussbezeichnungen (z.B. Realschulabschluss, Fachschulreife) zu vermeiden, sofern sich diese nicht zwingend aus den ausländischen Begriffen ableiten. Das gilt sinngemäß auch für Übersetzungen in eine fremde Sprache. (BDÜ, Landesverband Bayern, 2012: online).

Ein Übersetzer würde einem Feststellungs- oder Genehmigungsverfahren in Deutschland zum Beispiel vorgreifen, wenn er das Abschlusszeugnis eines mexikanischen „bachillerato“ oder einer „preparatoria“ mit „Abiturzeugnis“ oder „Allgemeine Hochschulreife“ übersetzt. Eine Abiturprüfung wie in Deutschland gibt es

${ }^{13}$ BDÜ = Bundesverband der Dolmetscher und Übersetzer; OMT = Organización Mexicana de Traductores. 
GRUHN, D. H. - Deutsch $\leftrightarrow$ Spanisch - Übersetzung

in Mexiko nicht und die Hochschulzugangsberechtigung müssen sich ausländische Studienbewerber in Deutschland u.U. erst durch den Besuch eines Studienkollegs erwerben.

Was die in der Leitlinie geforderte wörtliche Übersetzung bei Schultypen, Noten und Berufsbezeichnungen betrifft, so können bei diesem Vorgehen inhaltsleere Lehnübersetzungen (s.o.) entstehen. Zu prüfen ist, ob deren Funktion erkennbar ist, oder ob sie möglicherweise zu falschen Annahmen führen. Wer in Mexiko zum Beispiel sein grundständiges Medizinstudium abgeschlossen hat, trägt den Titel „médico-cirujano“, was nichts mit einem Fachstudium in Chirurgie zu tun hat. Es empfiehlt sich, den Begriff „Chirurg“ bei der Berufsbezeichnung im Deutschen $\mathrm{zu}$ vermeiden, und die Doppelbezeichnung in Klammern hinzuzufügen oder (besser) in einer Fußnote zu erklären.

\section{Eine Systematik der Begriffsäquivalenz}

Die eingangs behauptete Unübersetzbarkeit von Begriffen des Bildungswesens, ist an dieser Stelle zumindest teilweise wieder zurückzunehmen. Keine Gesellschaft kommt ohne ein wie auch immer geartetes Bildungswesen aus. Der Übersetzer steht also i.d.R. nicht vor völlig inkompatiblen Welten. Und das bedeutet, dass das System der Ausgangsgesellschaft für die Adressaten des ZT i.d.R. mithilfe von Begriffen der Zielgesellschaft verständlich gemacht werden kann. Doch wie verhalten sich die Begriffe von AS und ZS zueinander, bzw. welche Fehler können bei der Übersetzung auftreten? Um diese Fragen zu durchleuchten, muss zunächst einmal festgehalten werden, was überhaupt unter einem Begriff zu verstehen ist: „Ein Begriff ist [...] eine Denkeinheit, welche die gemeinsamen für den betreffenden Gegenstand relevanten Merkmale zusammenfasst. Die Auswahl dieser Merkmale kann nicht willkürlich sein, da sonst die fachliche präzise Kommunikation nicht möglich wäre“, heißt es bei Stolze (2013: 68; in Anlehnung an die DIN-Normen 2330 - 2333 und 2342 zur Begriffsbildung). Und wie kommen die Wörter zu den gedanklichen Begriffen? Stolze (ebd. 71f.) erklärt das folgendermaßen: „Die Begriffe werden mit [...] Benennungen, den Termini, bezeichnet. Dem Begriffssystem wird ein Benennungssystem zugeordnet. [...] Eine Benennung ist die mindestens ein Wort umfassende Bezeichnung eines Begriffs“. Der nächste Schritt besteht also darin, das Verhältnis zweier Begriffe der AS und ZS zueinander zu 
GRUHN, D. H. - Deutsch $\leftrightarrow$ Spanisch - Übersetzuns

bestimmen: „Sind die Begriffe, die durch Benennungen repräsentiert werden, in ihren Merkmalen gleich (begriffliche Identität), so sind auch die betreffenden Benennungen [...] äquivalent (wenn es sich um Benennungen verschiedener Sprachen handelt),“ schreiben Arntz, Picht und Schmitz (2014: 58 diesbezüglich.)

Äquivalenzmodelle haben versucht, das Verhältnis von (potenziell) vergleichbaren Begriffen in unterschiedlichen Sprachen zu beschreiben. Gemeinsamer Ausgangspunkt ist das klassische komparatistische Modell der Leipziger Schule. Otto Kade (1968, vgl. STOLZE 2011: 53) veranschlagt vier Arten ,potenzieller Äquivalente، zwischen Einzelsprachen, Dieses und weitere Nachfolgemodelle lassen sich in drei Grundfälle der denotativen Äquivalenz (je nach Autor mit weiteren Untergliederungen) zusammenfassen: „1. Die Begriffsinhalte decken sich vollständig, 2) teilweise, 3) nicht“ (STOLZE 2013: 74).

Bernard Thiry (2008) stellt diese Dreiteilung auf Grundlagen der Begriffstheorie. Als Autor eines zweisprachigen juristischen Wörterbuches Französisch $\leftrightarrow$ Spanisch (2005) beschäftigt er sich vorrangig mit juristischer Terminologie des Gerichtswesens. Eine (vereinfachte) Übernahme seiner Systematik für den hiesigen Kontext des Bildungswesens wird für die vorliegende Arbeit als zweckmäßig erachtet, da es sich bei beiden Systemen um präskriptive Begriffssysteme mit organisatorischer Funktion handelt. Seine Unterkategorien im Rahmen der Dreiteilung werden im folgenden Kapitel anhand von Beispielen aufgeführt.

Wie eingangs schon festgehalten, sind Begrifflichkeiten des Bildungswesens an ganz bestimmte Gesellschaften und Bildungssysteme gebunden. Aufgrund geschichtlicher Entwicklungen in den einzelnen Ländern und teilweiser Übernahme von Systemen anderer Staaten existieren allerdings auch viele Parallelen. Das umso mehr, als in den letzten Jahrzehnten versucht wird, nationale Bildungssysteme vergleichbar zu machen und somit aneinander anzupassen.

Auch wenn die in diesem Text als Beispiele verwendeten Begriffe isoliert dargestellt sind, soll das nicht darüber hinwegtäuschen, dass deren endgültige inhaltliche Bestimmung im konkreten Fall auch von textinternen und textexternen Zusammenhängen abhängt, die durchweg im Blickfeld bleiben müssen. Neben der denotativen Äquivalenz werden weitere Äquivalenztypen unterschieden (vgl. KOLLER 2011), hier ist vor allem die pragmatische Äquivalenz von Bedeutung, d.h. die Übersetzung muss für den 
GRUHN, D. H. - Deutsch $\leftrightarrow$ Spanisch - Übersetzung

Empfänger verständlich sein. An übergeordneter Stelle bei der Wahl einer geeigneten Übersetzungsstrategie steht somit die Funktion des jeweiligen Begriffs im Zieltext.

\section{Beispiele Deutsch $\leftrightarrow$ Spanisch anhand der Äquivalenz-}

\section{systematik}

\subsection{Vollständige Äquivalenz}

Ist eine vollständige begriffliche Äquivalenz gegeben, kann der Terminus der AS durch einen anderen in der ZS übersetzt werden, ohne dass erklärende Zusätze notwendig wären. Laut Thiry ist das dann gegeben, wenn die beiden dazugehörigen Begriffe sich in ihren semantischen bzw. inhaltlichen Merkmalen entsprechen. Anzumerken ist, dass die Termini beider Sprachen demselben Unterbereich des Zielsystems, hier also des Bildungswesens zugehörig sein müssen. Diese vollkommene Äquivalenz geht häufig mit einer wörtlichen Übersetzung bzw. morphologischen Entsprechung in beiden Sprachen einher (vgl. THIRY 2008: 531).

Tatsächlich ist das bei den hier behandelten Termini des Bildungswesens mehrheitlich der Fall. Würde man jeden Terminus erläuternd übersetzen oder eine Erklärung einfügen wollen, wäre der ZT kaum noch lesbar.

Ein typischer Fehler in diesem Zusammenhang ist die Nicht-Erkennung der Falschen Freunde. Von Wortpaaren, die sich in AS und ZS stark ähneln, wird fälschlicherweise angenommen, dass sie sich inhaltlich exakt entsprächen. Ein ,(alumno) desertor"14 zum Beispiel ist im Deutschen kein Deserteur, sondern ein Schul- oder Studienabbrecher. Da Sprachen die Welt unterschiedlich strukturieren, decken viele Begriffspaare gar nicht oder - häufiger noch - nur zum Teil semantisch vergleichbare Inhalte ab. Ein ungeübter Umgang mit zweisprachigen Wörterbüchern kann dabei zur Falle werden. Der mexikanische Terminus „estudiante“ kann auf Deutsch sowohl einen „Studenten“ als auch einen „Schüler“ bezeichnen und ist nur aus dem Textzusammenhang heraus korrekt interpretierbar.

Thiry (ebd.) unterscheidet bei der vollständigen Äquivalenz zwischen wörtlicher und nicht-wörtlicher Äquivalenz:

$14 \quad$ Vgl. den entsprechenden Eintrag in: http://dsia.uv.mx/cuestionario911/material_ apoyo/glosario\%20911.pdf. 
a) Wörtliche, morphologische Äquivalenz

Eine solche ist u.a. bei vielen Schul- und Studienfächern gegeben. Oft haben sie gemeinsame etymologische (z.B. lateinische) Wurzeln oder bezeichnen internationale Wissensgebiete. Beispiele wären Mathematik, Biologie, Anatomie usw. Die Übersetzung des Faches „Biologie“ mit „Biología“ oder umgekehrt bedeutet natürlich nicht, dass es sich dabei auch um dieselben Lehrinhalte handelt (falls der Adressat des ZT diesbezüglich Auskunft benötigt, wird er Lehr- oder Studienpläne anfordern).

Bei den vor allem in den Ingenieurwissenschaften und der Betriebswirtschaft (in Deutschland und in Mexiko) in Zeugnissen häufig verwendeten Anglizismen - manchmal auch nur als Akronym aufgeführt, z.B. TQM (Total quality management) - ist abzuwägen, ob der Adressat des ZT ein Fachmann ist. Das wird (gemäß des obigen Adressatenprofils der ZT) in der Regel nicht der Fall sein, weshalb eine (ausgeschriebene) Übersetzung in Klammern sich anbietet.

Des Weiteren fallen Eigennamen unter die Kategorie der wörtlichen Äquivalenz (Mexiko-Stadt, Fráncfort del Meno). Diese werden unverändert beibehalten, außer wenn es bereits eine allgemein übliche Bezeichnung in der Zielsprache gibt (vgl. z.B. FREIE und Hansestadt Hamburg 2010: 3f.). Ebenso verhält es sich bei kommerziellen Handelsnamen. Denn wer würde erahnen, auf was sich ein Kurs in „Tafel“ oder „pizarrón“ (Blackboard) bezieht?

Auch nicht sehr spezifische Hyperonyme haben meist eine wörtliche Äquivalenz, z.B. sistema educativo - Erziehungssystem / Bildungswesen; Schule - escuela; Universität - universidad.

Die morphologische Übereinstimmung zweier Termini kann allerdings auch zu Fehlschlüssen verleiten. Mexikanische „créditos“ können z.B. als Leistungspunkte oder durch den allgemein bekannten Anglizismus „credits“ übersetzt werden, nicht aber als Euro-Credits (ECTS), da Mexiko diesem System bisher nicht angeschlossen ist. Übersetzt man wiederum deutsche ECTS ins Spanische, so ist der Originalbegriff beizubehalten und inhaltlich zu erläutern. Ein weiteres Beispiel sind Wörter im Umfeld von „Diplom“. Handelt es sich um das Abschlussdiplom eines Schulungskurses, so werden „Diplom“ und „diploma“ i.d.R. Äquivalente sein. Ein Diplom-Abschluss hingegen ist etwas völlig anderes (nämlich ein traditionsreicher deutscher Studienabschluss, der auch Zugang zur 
GRUHN, D. H. - Deutsch $\leftrightarrow$ Spanisch - Übersetzuns

Promotion verschafft ${ }^{15}$ ), was auf jeden Fall in einer Fußnote erläutert werden muss und nicht mit „diploma“ zu übersetzen ist. Andererseits gibt es in Mexiko „diplomados“, das sind (meist) universitäre Fortbildungskurse, die sich über rund 120 Unterrichtsstunden erstrecken, und für deren Abschluss man am Ende ein „diploma“ erhält, das wiederum mit einem „Diplom-Abschluss“ nichts zu tun hat.

b) Nicht wörtliche Äquivalenz

Auch in diesem Fall lässt sich ein Terminus der AS problemlos in einen anderen der ZS überführen, der aber morphologisch nicht oder nur in Teilen verwandt ist bzw. dessen Wortlaut keine wörtliche Übersetzung darstellt. Beispiele wären z.B. die Übersetzung des Faches „asignatura estatal“ als „Staatsbürgerkunde“ oder „apreciación artística“ als „Kunst“, des Studiengangs „ingeniería mecatrónica“ als „Mechatronik“ (unter Auslassung des ersten Teils, d.h. „ingeniería“) oder „ingeniería de procesos“ als „Verfahrenstechnik“ in Zeugnissen. Ein „examen profesional“ bei grundständigen Studiengängen (Licenciatura) ist die „mündliche Verteidigung der Abschlussarbeit“, „sinodales“ sind „Mitglieder eines Prüfungsausschusses bzw. einer Prüfungskommission“. Ein „pasante“ ist ein Studienabsolvent ohne Titel. Wobei die Kompaktheit des mexikanischen Terminus wohl darauf zurückzuführen ist, dass solche „pasantes“ in Mexiko häufig sind und bis vor wenigen Jahren auf dem Arbeitsmarkt gute Chancen hatten. Sie haben sämtliche Studienfächer abgelegt, bringen aber ihre schriftliche Abschlussarbeit nicht bzw. nie zu Ende (weshalb man nun weitere Optionen des Titelerhalts eingeführt hat, die von der leidlichen Abschlussarbeit befreien). Im konkreten Fall ist zu prüfen, ob es sich um einen Studienabsolventen handelt, der noch an seiner Abschlussarbeit schreibt, oder ob „Studienabbrecher“ die korrekte Übersetzung wäre.

Ein bestimmter Wortstamm impliziert nicht zwangsweise, dass bei der Übersetzung der in der ZS entsprechende Stamm zu verwenden wäre. So haben z.B. Wortbildungen mit „profesion“ zwar immer etwas mit Beruf oder fachlichem Können zu tun (profesional/professionell). Ein „profesionista“ ist aber in der Fachsprache - anders

\footnotetext{
15 Seit dem Bologna-Prozess schien der deutsche Diplomstudiengang ein Auslaufmodell zu sein. Tatsächlich wird er aber an einigen Universitäten beibehalten oder sogar wieder neu eingeführt. Vgl. die folgende Publikation des Vereins deutscher Ingenieure (VDI): https://www.ingenieur.de/karriere/bildung/hochschule/diplom-ingenieur-studiengaenge/ (Stand 11.02.2021).
} 
GRUHN, D. H. - Deutsch $\leftrightarrow$ Spanisch - Übersetzung

als manche Wörterbücher vorgeben - nicht Fachmann irgendeines Bereiches, sondern ein Akademiker (vgl. InSTITUTO Nacional de Estadística, Geografía e Informática o.J.).

\subsection{Teiläquivalenz}

Die semantischen bzw. inhaltlichen Merkmale der jeweiligen Begriffe in AS und ZS überlappen sich zwar teilweise, sind aber nicht vollkommen deckungsgleich. D.h. die zu vergleichenden Begriffe umfassen in einer oder beiden Sprachen zusätzliche inhaltliche Merkmale oder Verwendungszusammenhänge, die in der anderen nicht gegeben sind. Nur dort, wo beide in AS und ZS übereinstimmen, d.h. dieselben Merkmale abdecken, kann das Wortpaar als äquivalent gewertet werden. Andernfalls ist in der ZS ein anderer Terminus zu wählen (vgl. ebd.: 532).

Hier liegt eine der gewichtigsten Fehlerquellen, da die abweichenden Begriffsmerkmale oftmals nicht erkannt werden. Das Phänomen betrifft allgemein die meisten in AS und ZS zu vergleichenden Begriffsbenennungen, einschließlich solcher „falschen Freunde“, deren inhaltliche Merkmale bzw. Verwendungskontexte (nur) teilweise übereinstimmen. Nachfolgend einige Beispiele:

\begin{tabular}{|l|l|l|}
\hline Deutsch & Spanisch & \multicolumn{1}{|c|}{ Kommentar } \\
\hline Akademie & Academia & $\begin{array}{l}\text { Kann in beiden Sprachen eine Institution bezeichnen, in Dtl. meist } \\
\text { auf hohem Niveau (z.B. Akademie der Wissenschaften), in } \\
\text { Mexiko meist Privatinstitutionen, die eine Fertigkeit lehren (z.B. } \\
\text { Academia de baile). } \\
\text { Weitere Bedeutung: Dtl.: Vereinigung von Gelehrten, Künstlern } \\
\text { oder Dichtern (vgl. Duden); Mex.: Lehrerkollegium eines } \\
\text { Fachbereichs } \\
\text { Nur in wenigen Fällen liegt eine vollständige Äquivalenz vor. } \\
\text { Eine „Academia de alemán“(Lehrerkollegium einer universitären } \\
\text { Deutschabteilung) (vgl. SEP o.J.) ist z.B. nicht als „Deutsch- } \\
\text { Akademie“ zu übersetzen. Der Terminus bezeichnet im } \\
\text { Deutschen mitunter auch Sprachschulen. } \\
\text { Das deutsche Adjektiv ,akademisch“ist an das Universitätswesen } \\
\text { gebunden, ,académico“ auf (mex.) Spanisch wird allgemein im } \\
\text { Umfeld der Lehre, also auch des Schulwesens verwendet. }\end{array}$ \\
\hline
\end{tabular}


GRUHN, D. H. - Deutsch $\leftrightarrow$ Spanisch - Übersetzuns

\begin{tabular}{|c|c|c|}
\hline Karriere & Carrera & $\begin{array}{l}\text { Die Termini können sich in beiden Sprachen auf eine berufliche } \\
\text { Karriere (also den beruflichen Aufstieg) beziehen. In Mexiko viel } \\
\text { häufiger ist aber die Bedeutung „Studiengang“, die „Karriere“ } \\
\text { nicht umfasst. }\end{array}$ \\
\hline Professor & Profesor & $\begin{array}{l}\text { Beide beziehen sich auf eine Lehrtätigkeit, weisen aber einen } \\
\text { unterschiedlichen Verwendungsumfang auf: In Mex. auf alle } \\
\text { Bildungsstufen bezogen, in Dtl. höchste Rangstufe an einer } \\
\text { Universität und zwangsweise mit Forschungstätigkeiten } \\
\text { verbunden. In Dtl. ist die Berufung eines Professors an das } \\
\text { staatliche Beamtensystem geknüpft, weshalb die Frage, ob } \\
\text { jemandem der Namenszusatz „Prof.“ rechtmäßig zusteht, } \\
\text { mitunter auch die Gerichte beschäftigt }{ }^{16} \text {. Schon aus diesem Grund } \\
\text { ist die Übersetzung von „profesor“ als „Professor“ besser zu } \\
\text { vermeiden. „Lehrer“ (im schulischen Kontext), } \\
\text { „Universitätsdozent“, „Hochschullehrer“ oder auch } \\
\text { „Fachbereichsleiter“o.Ä. bieten sich an. }\end{array}$ \\
\hline $\begin{array}{l}\text { Technik / } \\
\text { Techniker }\end{array}$ & $\begin{array}{l}\text { Técnica / } \\
\text { técnico }\end{array}$ & $\begin{array}{l}\text { Die Termini beider Sprachen können sich auf technische Prozesse } \\
\text { beziehen, aber auch auf eine bestimmte Vorgehensweise oder } \\
\text { Methode. Handelt es sich jedoch um Fachbereiche der Technik, } \\
\text { steht im mex. Spanisch häufig „,ingeniería“. Aus } \\
\text { Verfahrenstechnik wird dann z.B. „ingeniería de } \\
\text { procedimientos“. Geht es um Personen, so kann ein } \\
\text { mexikanischer „técnico“ ein Techniker sein, meist wird damit } \\
\text { aber ein Fachmann ohne Universitätsstudium bezeichnet. Als } \\
\text { Adjektiv wird „(nivel) técnico“ für nicht akademische Berufe } \\
\text { bzw. Berufsausbildungen (aller Art, d.h. nicht nur technischer } \\
\text { Inhalte) verwendet. }\end{array}$ \\
\hline
\end{tabular}

Quelle: eigene Darstellung

\subsection{Nicht-Äquivalenz}

Im Falle der völligen Nicht- Äquivalenz, d.h. der Nicht-Existenz eines entsprechenden Begriffs der AS in der ZS unterscheidet Thiry drei Fälle: a) Weder ein Begriff noch ein Terminus existieren in der ZS. b) Der Begriff existiert in der ZS, aber es gibt keinen

\footnotetext{
${ }^{16}$ Auf Grundlage von § 132a des deutschen Strafgesetzbuches (https://dejure.org/gesetze/StGB/132a.html).
} 
GRUHN, D. H. - Deutsch $\leftrightarrow$ Spanisch - Übersetzung

dazugehörigen Terminus. c) Es existiert ein Terminus in der ZS, doch der Begriff existiert nicht (ebd.: 535).

Häufigster Übersetzungsfehler im Falle der Nicht-Existenz einer Äquivalenz ist, dass diese nicht als solche erkannt und durch einen vermeintlich äquivalenten Terminus in der ZS ersetzt wird (z.B. „bachillerato“ durch „Abitur“, „Gymnasium“ durch „bachillerato“).

$\mathrm{Zu}$ a): Für den Fall, dass weder ein Begriff noch ein Terminus in der ZS existieren, schlägt Thiry vor, Neologismen $\mathrm{zu}$ bilden. Das ist aber im Hinblick auf die Urkundenübersetzung selten von Vorteil, da solche Neologismen für den Adressaten der Übersetzung i.d.R. nicht verständlich wären. Die Vorgehensweise des Übersetzers wird in der Beibehaltung der Bezeichnung in der Originalsprache bestehen, zuzüglich einer erklärenden Fußnote. Denn der „städtischen schulunabhängigen Orientierungsstufe“ (München) oder dem „Lobo-Mentor” (Puebla ${ }^{17}$ ) könnte ein Neologismus nicht gerecht werden.

Einen Sonderfall stellen die oben schon angesprochenen inhaltsleeren Lehnübersetzungen dar, die infolge von wörtlichen Übertragungen entstehen (Amtsbezeichnung von Unterzeichnern von Schulzeugnissen z.B.). Auch hier gibt es weder Begriff noch Terminus in Zielgesellschaft bzw. ZS. Doch wird aus der wörtlichen Übersetzung im textinternen Kontext die Funktion der sprachlichen Schöpfung erkenntlich, was in diesem Fall ausreichend ist.

$\mathrm{Zu}$ b): Der zweite Fall wird für diese Arbeit dahingehend abgeändert, dass Begriffe der Ausgangsgesellschaft in den Fokus genommen werden, für die in der ZS kein Terminus existiert. Auch der Begriff existiert im Bildungssystem der Zielgesellschaft nicht, er wird aber im Kontext durch eine wörtliche Übersetzung, eine Lehnübersetzung oder eine beschreibende Übersetzung unmittelbar einsichtig, ohne dass eine erläuternde Fußnote notwendig wäre. Dieser Fall ist häufig, er trifft zum Beispiel auf „exotische“ Schulfächer zu, die auch in der Ausgangsgesellschaft meist Schöpfungen einzelner Schulen sind: ein Fach mit Namen „Sentido de la vida“ oder, in umgekehrter Richtung, „Glück“ (es gibt das wirklich) werden als „Sinn des Lebens“ bzw. „Felicidad“ für den Adressaten verständlich sein (wenn er auch rätseln mag, was sich dahinter

\footnotetext{
${ }^{17}$ Es handelt sich um Studenten einer öffentlichen Universität in Puebla, die neu aufgenommenen jüngeren Studentengenerationen beratend zur Seite stehen. Der Zusatz "lobo" (Wolf) ist dabei von der einst zur Universität gehörenden Fußballmannschaft (Lobos BUAP) entlehnt.
} 
GRUHN, D. H. - Deutsch $\leftrightarrow$ Spanisch - Übersetzung

verbirgt). Das Schulfach „Colotli ${ }^{18 ،}$ kann als „Entwicklung der Denkfähigkeiten“ umschrieben werden. Eine etymologische Erklärung ist für die Adressaten der Übersetzung irrelevant (d.h. aus funktionaler Perspektive nicht notwendig).

Weitere Beispiele wären die ,cédula profesional“" die in Mexiko alle Akademiker mit Abschlusstitel besitzen. In Deutschland ist ein gesonderter Ausweis neben dem Titel nur bei wenigen Berufsgruppen üblich. Eine wörtliche Übersetzung (z.B. Berufsausweis) wird aber verständlich sein (die Funktion des Begriffes im Kontext ist abzugleichen). Die „educación básica“ wiederum umfasst in Mexiko den „Elementar-, Primar- und Sekundarbereich bis einschließlich der 9. Jahrgangsstufe“" und kann ggf. auch so übersetzt werden.

Zu c): Der dritte Fall wird im Rahmen der Urkundenübersetzung kaum jemals auftreten, weshalb er hier ausgeschlossen wird. Denkbar sind lediglich aus anderen Sprachen übernommene Termini in der ZS, z.B. für neu geschaffene Studien- und Forschungsrichtungen oder auch bildungspolitische Konzepte die aber im Bildungssystem der Zielgesellschaft (noch) nicht existieren. So wurde in Mexiko z.B. von “competencias" gesprochen, noch bevor der Begriff inhaltlich in Bildungsplänen usw. verankert war.

\section{Inhaltliche Bestimmung der Begriffe}

Im Arbeitsalltag wird der (immer unter Zeitdruck arbeitende) professionelle Übersetzer Entscheidungen aufgrund seines Fach- und Allgemeinwissens sowie seiner sprachlichen Kompetenz und Erfahrung treffen. Entzieht sich ein Begriff dieser Zugangsweise, sind Hilfsmittel heranzuziehen.

\subsection{Gängige Hilfsmittel}

„Der Hochschulwortschatz ist in allgemeinsprachigen Wörterbüchern entweder gar nicht oder nur unzureichend vorhanden“, schreibt Reichmann (2013), wobei sie die einsprachigen Wörterbücher Duden (2007) und Wahrig (2005) als Beispiele anführt. „Auch in zweisprachigen Wörterbüchern Deutsch/Portugiesisch [...] sind keine qualitativ

\footnotetext{
${ }^{18}$ Von náhuatl „colotli“: In prähispanischen Gesellschaften ein hölzernes Gittergestell, das als Unterbau für eine Götterfigur diente (vgl. UNIVERSIDAD Nacional Autónoma de México 2012). Dieses durchlässige Fundament wird als Bildnis für den offenen, empfangenden menschlichen Geist genommen.
} 
GRUHN, D. H. - Deutsch $\leftrightarrow$ Spanisch - Übersetzung

hochwertigen Übersetzungslösungen zu erwarten“, konstatiert sie (ebd.). Das gilt analog für das Sprachenpaar Deutsch $\leftrightarrow$ Spanisch. Solche zweisprachigen Wörterbücher bergen im Übrigen Gefahren. Zum einen, weil die meisten keine Definitionen und Verwendungskontexte angeben. Zum anderen, weil sie i.d.R. nicht länderspezifisch konzipiert sind. Das für Stipendiaten und Gastwissenschaftler vom DAAD erstellte Wörterbuch zu Begriffen aus Wissenschaft und Hochschule Deutsch - Spanisch (DAAD 1998) ist zum Beispiel u.a. aus eben diesen Gründen für Übersetzer ungeeignet ${ }^{19}$.

Idealerweise bedürfte es doppelt einsprachiger Fachwörterbücher, die zwischen verschiedenen nationalen Bildungssystemen mit derselben Standardsprache unterscheiden.

In Bezug auf das deutsche Bildungssystem kann das von der Europäischen Kommission herausgegebene und kostenlos im Internet verfügbare Europäische Glossar zum Bildungswesen eine Stütze $\operatorname{sein}^{20}$. Zwischen 1999 und 2008 erschienen davon insgesamt fünf Bände zu verschiedenen Themenbereichen (Bildungseinrichtungen, Lehrpersonal u.a.). Nachteil der Reihe ist, dass sie nicht diachronisch konzipiert und seit 2008 nicht aktualisiert worden ist. Nationale Bildungsysteme sind häufigen Reformen unterworfen und somit auch Veränderungen ihrer Begriffssysteme.

Ständig aktualisiert wird die Webseite der Kultusministerkonferenz (2010-2021), hier ist insbesondere der Link zur Zentralstelle für Ausländisches Bildungswesen (ZAB) von Bedeutung, über den auch summarische Übersichten über die einzelnen nationalen Bildungssysteme und -abschlüsse abrufbar sind. Von dort gelangt man auf das Infoportal zu ausländischen Bildungsabschlüssen (anabin), das auch ein (mehrsprachiges) Glossar enthält. Dass aber kein Glossar den Übersetzer davon befreit, weitere Quellen heranzuziehen oder gegebenenfalls eigene Kriterien anzulegen, zeigt ein Beispiel aus dem letztgenannten in Kapitel 7 dieses Artikels.

Bezüglich der Fachtermini des mexikanischen Bildungswesens gibt es kein umfassendes Verzeichnis. Im Internet kursieren einige (einsprachige) online-Glossare, die sich auf Ausschnitte des Systems beziehen, meist sehr allgemein gehalten sind und

\footnotetext{
${ }^{19}$ Viele der dort aufgeführten span. Termini sind in Mexiko ungebräuchlich (z.B. wurden technische Studienfächer meist als „técnica ins Spanische“ übersetzt - „electrotecnia“, statt „,ingeniería eléctrica“), manche schwer verständlich (z.B. „Einstufungstest“: „test de clasificación“", statt „examen de ubicación“) oder völlig unverständlich (z.B. „Einwohnermeldeamt“: „oficina de empadronamiento“; „,catedrático supernumerario“: „Außerordentlicher Professor“). Auch enthält es einige Fehler (z.B.: „,bachillerato: „Abitur/Allgemeine Hochschulreife“; „Handapparat [i.e.Bibliothek]“: „obras de referencia)“".

${ }^{20}$ Dieses und weitere Glossare zum europäischen Bildungswesen können dem Deutschen Bildungsserver entnommen werden: https://www.bildungsserver.de/Glossare-zum-Bildungswesen-2142-de.html.
} 
GRUHN, D. H. - Deutsch $\leftrightarrow$ Spanisch - Übersetzuns

darüber hinaus kaum jemals aktualisiert werden. Oftmals enthalten sie auch keine Angabe zu Autoren oder Erscheinungsdatum (z.B. SEP o.J.; SEP 2008).

Regelmäßig wird der Urkundenübersetzer auf (einsprachige) normative Dokumente der nationalen bzw. bundesstaatlichen Regierungen bzw. derer Bildungsbehörden und Zusammenschlüsse im Bereich des Schul- oder Universitätswesens $^{21}$ oder einzelner Institutionen zurückgreifen. Darunter fallen z.B. Schul- oder Hochschulgesetze, Lehr- und Studienpläne, offizielle Statistiken u.a. Mitunter helfen auch akademische Schriften weiter. Wenn sich auch dort keine klare Definition oder Bestimmung finden lässt, was relativ häufig der Fall ist, muss selbständig eine Merkmalbestimmung durchgeführt werden.

\subsection{Merkmalbestimmung}

„Unter dem 'Umfang eines Begriffs' versteht man die Gesamtheit aller individuellen Gegenstände, die sämtliche Merkmale des Begriffs aufweisen“ heißt es bei Stolze (2013: 68f., wobei sie sich auf die DIN-Norm 2330 bezieht). „Die Gesamtheit der zu einem gegebenen Zeitpunkt festgestellten Merkmale eines Begriffs ist die Summe des Wissens über einen Begriff“ schreiben Arntz, Picht und Schmitz (2014: 57). Merkmale können nach verschiedenen Kriterien unterteilt werden, so nach Beschaffenheits- und Relationsmerkmalen (vgl. ebd.: 59), aber auch nach „wesentlichen” und "unwesentlichen", doch ,'Wesentlichkeit' ist [...] eine relative Größe, d.h., dass man nur in einer gegebenen Situation entscheiden kann, ob ein Merkmal wesentlich ist oder nicht", (ebd.: 61). Im hier behandelten Zusammenhang sind Merkmale dann wesentlich, wenn sie einen Einfluss auf die Funktion des Translats haben, d.h. wenn sie die Entscheidungen des Adressaten (z.B. eines Sachbearbeiters) beeinflussen können.

Praktizierende Übersetzer sind i.d.R. keine Fachterminologen. Um sich an die Merkmale einzelner Begriffe anzunähern, werden sie Sekundärquellen heranziehen oder Fachleute befragen (die sich allerdings untereinander nicht immer einig sind). Dabei muss bei Nicht- oder Teil Äquivalenz keine vollständige Merkmalsbestimmung durchgeführt werden, vielmehr hat diese ihren Zweck erfüllt und kann abgebrochen werden, sobald

21 Deutschland: Kultusministerkonferenz (KMK), Hochschulrektorenkonferenz (HRK); Mexiko: Asociación Nacional de Universidades e Instituciones de Educación Superior (ANUIES), das Schulwesen ist zentralstaatlich geregelt (SEP), mit Delegationen und eigenem Regierungssekretär in den einzelnen Staaten. Einen Zusammenschluss der einzelstaatlichen Autoritäten gibt es nicht. 
GRUHN, D. H. - Deutsch $\leftrightarrow$ Spanisch - Übersetzung

deutlich wird, welche Übersetzungsstrategie in Hinsicht auf die Funktion des Begriffes im ZT zu wählen ist.

Nachfolgend eine Übersicht anhand bereits vorgestellter Beispiele über das (recht überschaubare) Instrumentarium der Begriffsübersetzung im Bereich des Bildungswesens:

\begin{tabular}{|c|c|}
\hline Übersetzungsstrategie & Beispiele \\
\hline $\begin{array}{l}\text { Übersetzung des Terminus der AS } \\
\text { durch einen diesem wörtlich oder } \\
\text { nicht wörtlich entsprechenden } \\
\text { Terminus in der ZS }\end{array}$ & $\begin{array}{l}\text { Anatomía - Anatomie } \\
\text { Profesionista - Akademiker }\end{array}$ \\
\hline $\begin{array}{l}\text { Lehnübersetzung (inhaltlich } \\
\text { verständlich oder nur von der } \\
\text { Funktion her verständlich) }\end{array}$ & $\begin{array}{l}\text { Cédula profesional - Berufsausweis } \\
\text { Studiendirektor - director de estudios }\end{array}$ \\
\hline Umschreibende Übersetzung & $\begin{array}{l}\text { Examen profesional - mündliche Verteidigung der } \\
\text { Abschlussarbeit (unterhalb des Doktorandenstudiums, } \\
\text { bei Promotion: Disputation) } \\
\text { Educación básica - Elementar-, Primar- und } \\
\text { Sekundarbereich bis einschließlich der } 9 . \\
\text { Jahrgangsstufe }\end{array}$ \\
\hline $\begin{array}{l}\text { Beibehaltung des Terminus der AS } \\
\text { in der ZS mit zusätzlicher } \\
\text { erklärender Übersetzung in } \\
\text { Klammern (bzw. umgekehrt, der } \\
\text { Terminus der AS steht in } \\
\text { Klammern) }\end{array}$ & $\begin{array}{l}\text { Diplomado - Universitärer Fortbildungskurs } \\
\text { (Diplomado) } \\
\text { Colotli - Entwicklung der Denkfähigkeiten (Colotli) }\end{array}$ \\
\hline $\begin{array}{l}\text { Beibehaltung des Terminus der AS } \\
\text { in der ZS und Anbringung einer } \\
\text { erklärenden Fußnote } \\
\text { (Wobei der Inhalt der Fußnote } \\
\text { jeweils vom der Funktion des } \\
\text { Begriffes in der ZS abhängt, d.h. es } \\
\text { müssen i.d.R. keine vollständigen }\end{array}$ & $\begin{array}{l}\text { 11. Klasse Gymnasium (Nota del traductor: El } \\
\text { „Gymnasium“ es un tipo de escuela del sistema } \\
\text { eductivo alemán que no tiene un equivalente exacto } \\
\text { en el sistema educativo mexicano. Comprende a lo } \\
\text { que en México serían los dos últimos grados de la } \\
\text { educación primaria, los tres grados de la educación } \\
\text { secundaria y la educación media superior. „11. }\end{array}$ \\
\hline
\end{tabular}


GRUHN, D. H. - Deutsch $\leftrightarrow$ Spanisch - Übersetzung

\begin{tabular}{|l|l|}
\hline $\begin{array}{l}\text { Beschreibungen oder Definitionen } \\
\text { vorgelegt werden.) }\end{array}$ & Klasse“ corresponde al segundo grado de la \\
& educación media superior. \\
& Médico-cirujano (Fußnote: Wörtlich: Arzt und \\
& Chirurg. Mexikanischer Universitätstitel, den der \\
& Absolvent eines grundständigen medizinischen \\
& Studiengangs erwirbt. ) \\
\hline
\end{tabular}

Beispiel: Ein Kunde (mit doppelter Staatsbürgerschaft) möchte sein Abschlusszeugnis der „Licenciatura en Enseñanza y Aprendizaje del Inglés en Educación (Schulstufe)“ einer Escuela Normal Superior in Mexiko als „Bachelor of Arts in Lehramt für Englisch“ übersetzt haben. Auf den ersten Blick scheint das plausibel, denn beide Benennungen verweisen auf einen grundständigen Studiengang, der auf eine Lehrtätigkeit an Schulen vorbereiten soll. Untersucht man die Zusammenhänge jedoch eingehender, wird schnell ersichtlich, dass eine Beschreibung der Studiengänge, die zur Lehre an Schulen ermächtigen, für beide Länder sehr komplex ist.

Als Ausgangssituation ist festzuhalten, dass die Originalzeugnisse auf einen sehr spezifischen Sachverhalt verweisen, d.h. auf einen in Studienplänen fest umrissenen Studiengang, der von einer konkreten Institution an einem bestimmten Ort und zu einem bestimmten Zeitpunkt angeboten wird. Das Translat hingegen muss (nicht nur in diesem Fall) i.d.R. in einem sehr breiten Rahmen interpretierbar sein. Es kann bei verschiedenen Institutionen oder Stellen (evtl. mehrerer Bundesländer), möglicherweise auch wiederholt $\mathrm{zu}$ unterschiedlichen Zeitpunkten eingereicht werden. Dabei ist denkbar, dass es zu unerschiedlichen Studienorganisationsformen der Zielgesellschaft in Vergleich gesetzt wird.

In Deutschland koexistieren infolge des Bologna-Prozesses seit dem ersten Jahrzehnt des 21. Jahrhunderts das herkömmliche Lehramtsstudium (mit zwei Staatsexamina) und ein zweistufiges Bachelor $^{22}$ - und Master-Modell. Da das Bildungswesen in Deutschland Ländersache ist, bieten einige Länder nur eines von beiden an, andere (noch) beide, vereinzelt existieren sogar weitere Ausbildungsformen (z.B. Pädagogische Hochschulen in Baden-Württemberg). Reformen können Status und

\footnotetext{
${ }^{22}$ Die einschlägigen in Deutschland angebotenen Studiengänge können über die Seite Hochschulkompass https://www.hochschulkompass.de/home.html (Stand 12.04.2021) eingesehen werden.
} 
GRUHN, D. H. - Deutsch $\leftrightarrow$ Spanisch - Übersetzuns

Inhalte von Bildungsgängen wesentlich verändern und somit auch die damit in Verbindung stehenden Begriffssysteme.

Selbst innerhalb der einzelstaatlichen Bildungssysteme können „Licenciatura“ oder „Bachelor“ bezüglich Dauer, Studienpläne, Anzahl der studierten Credits usw., aber auch je nach Zeitraum des Studiums sehr Unterschiedliches beinhalten. Solange es kein bilaterales Gleichstellungsabkommen gibt, ist auch bei Anerkennungsverfahren nicht grundsätzlich von einer (gegenseitigen) Anerkennung auf der entsprechenden Stufe auszugehen. $\mathrm{Zu}$ bedenken ist weiterhin, dass das unbefugte Führen inländischer akademischer Grade nach § 132a StGB in Deutschland unter Strafe steht.

Der Versuch, Lösungsansätze im Glossar der Datenbank anabin (KULTUSMINISTERKONFERENZ 2010-2021) zu finden, führt nicht weiter. Für das direkt auf Mexiko bezogene Lemma „Licenciatura“ erscheint dort die in Deutschland kaum gebräuchliche ${ }^{23}$, aus dem Mittelalter stammende „Lizentiatur“.

Ganz im Sinne der in Kapitel 4 dieser Arbeit zitierten Leitlinie, bleibt als Überstzungsstrategie für „Licenciatura“ lediglich die Beibehaltung der Originalbezeichnung und die Erläuterung des Begriffs in einer Fußnote (grundständiger Studiengang in Mexiko).

Wie verhält es sich nun mit dem Begriff des „Lehramts“? Die traditionsreiche mexikanische Escuela Normal Superior ist nur eine von mehreren Möglichkeiten, sich in Mexiko als Lehrer auszubilden, sie umfasst ein landesweites Netz öffentlicher und privater Einrichtungen unter staatlicher Kontrolle. Fokus der Ausbildung ist die Lehre an Schulen der „educación básica“ (das umfasst die seit 2008 obligatorischen Vorschule ${ }^{24}$ bis einschließlich der 9. Jahrgangsstufe). Erst seit 1984 sind ihre Abschlüsse als Hochschulabschlüsse anerkannt (vgl. PERFILES EDUCATIVOs 2018). Und bis zum Jahr 2016 war es ausschließlich ihren Absolventen möglich, sich über Ausschreibungsverfahren für den staatlichen Schuldienst zu bewerben (vgl. SEP 2016), Absolventen anderweitiger Lehrerstudiengänge konnten lediglich an Privatschulen unterrichten.

\footnotetext{
${ }^{23}$ In einigen Fachbereichen wird der Titel Lizenziat noch vergeben, z.B. im katholischen Hochschulrecht, wo es einem Doktorgrad gleichgestellt ist (vgl. z.B. https://www.unimuenster.de/FB2/ikr/lizenziat/index.shtml, Stand 11.04.2021).

${ }^{24}$ Diario Oficial de la Federación (Regierungsanzeiger) vom 12. November 2002. Online: https://www.scjn. gob.mx/sites/default/files/cpeum/decretos_reformas/2016-12/00130170.pdf (Stand: 15.04.2021).
} 


$$
\text { GRUHN, D. H. - Deutsch } \leftrightarrow \text { Spanisch - Übersetzung }
$$

In Deutschland war die Lehre an öffentlichen Schulen bis zur Jahrtausendwende relativ fest an den Beamtenstatus geknüpft: „Da der Schulunterricht als 'Ausübung hoheitlicher Rechte' Angehörigen des öffentichen Dienstes zu übertragen ist [...], müssen nach der vorherrschenden Überzeugung Lehrer in der Bundesrepublik in der Regel Beamte sein“ (ARBEITSGRUPPE Bildungsbericht am Max-Planck-Insitut für Bildungsforschung 1994: 105). Insofern war das Bestimmungswort „Amt“ des Kompositums „Lehramt“ im Sinne des Beamtenrechts zu verstehen (vgl. das Lemma „Amt“ im Duden Recht A-Z 2015). Gegenwärtig erfolgt die Einstellung an Schulen häufig auch ohne Verbeamtung. Und infolge der Bologna-Reformen gibt es nun Bachelor-Sudiengänge in Lehramt ${ }^{25}$, die es Absolventen i.d.R. (d.h. je nach Bundesstaat und Bildungsbereich) gar nicht ermöglichen, sich als Staatsbeamter zu bewerben, d.h. ein solches Lehramt überhaupt anzutreten. Dazu ist der Abschluss auf der Stufe eines Aufbaustudiengangs (Master, Zweites Staatsexamen) zu erwerben.

Die Rechtsbestimmungen bezüglich des Zugangs zu einem Lehramt sind also nicht nur im Vergleich der einzelnen Bundesländer innerhalb Deutschlands heterogen, sondern unterscheiden sich auch wesentlich im deutsch-mexikanischen Vergleich und gemäß ihrer zeitlichen Verortung. Betrachtet man darüber hinaus inhaltliche Aspekte der Studiengänge beider Länder, ein Lehramtsstudium in Deutschland umfasst i.d.R. zwei fachliche Schwerpunkte und ein zweijähriges Referendariat, das Studium an der Escuela Normal lediglich ein Schulfach und nur 16 Wochen Praktikum, sind die zusammengetragenen Daten hinreichend, um deutlich zu machen, dass eine Übersetzung als „Lehramt“ einem behördlichen Feststellungs- oder Anerkennungsverfahren vorgreifen würde. Als Übersetzung bietet sich z.B. „Licenciatura* in Lehr- und Lernprozessen des Englischen" an (das * verweist auf eine Fußnote). So ist die Fachrichtung des Studiums umrissen, ohne dass eine Gleichstellung mit einem deutschen Lehramtsstudium suggeriert wird.

\section{Begriffe des Bildungswesens in akademischen Texten}

Es stellt sich die Frage, ob die in diesem Artikel für die Urkundenübersetzung vorgestellten Überlegungen nicht auch auf andere Textsorten übertragbar sind. Was

\footnotetext{
${ }^{25}$ Der Name der Studiengänge enthält häufig den Begriff „Lehramt“, die dabei erworbenen Abschlusstitel „Bachelor in Education“" allerdings nicht.
} 
GRUHN, D. H. - Deutsch $\leftrightarrow$ Spanisch - Übersetzung

akademische Texte und Texte im akademischen Umfeld $^{26}$ betrifft, die sich mit bildungswissenschaftlichen Aspekten auseinandersetzen, ist das ausdrücklich zu bejahen. Dabei handelt es sich nicht unbedingt um vollständig übersetzte Texte, sondern häufig nur um die Übersetzung einzelner Termini.

Die Übersetzungssituation unterscheidet sich bei beiden Textsorten wesentlich. Die Adressaten akademischer Texte, die im Rahmen von Forschungsarbeiten entstehen, sind i.d.R. keine Beamte, sondern Akademiker, AT und ZT sollen nicht behördlichen Zwecken, sondern wissenschaftlichem Erkenntnisgewinn dienen. Doch gibt es auch verbindende Elemente. Bildungssysteme sind öffentlich-rechtliche Systeme, die durch spezifische (nicht immer eindeutig definierte) Begriffe beschrieben werden. Von Wissenschaftlern wird verlangt, ihr jeweiliges Begriffsverständnis auszuführen, wissenschaftliche Sachverhalte sollen objektiv und trennscharf dargestellt werden.

Es gilt auch hier der Grundsatz, den Stolze (2013: 285) für die Urkundenübersetzung formuliert: „Nicht das Vermeiden unüblicher Formulierungen oder unspezifischer Ausdrücke, sondern von Falschaussagen ist [...] wesentlich“. Wird also in einem Fachtext eine vermeintliche Äquivalenz zwischen zwei Termini veranschlagt, ohne sie zu sachgerecht zu hinterfragen, kann es zu eben solchen Falschaussagen kommen. Dabei ist - ebenso wie im Falle der Urkunden - auf den zeitlichen Kontext zu achten, in den ein bestimmter Begriff gestellt wird: „Vielfach ist das Fortbestehen einer Benennung kein sicherer Indikator dafür, dass auch der Begriff der gleiche geblieben ist, z.B. hat sich die wissenschaftliche Betrachtung des Atoms [...] entscheidend geändert, ohne dass deswegen die Benennung geändert worden wäre“ (ARNTZ; PICHT; SCHMITZ 2014: 58). Im bildungswissenschaftlichen Bereich wäre diesbezüglich an die Ernennung von Professoren in Deutschland zu denken, für die ab 2003 mit einer Änderung des Hochschulrahmengesetzes und der Einführung von Juniorprofessuren nicht mehr zwingend eine Habilitation notwendig ist ${ }^{27}$, aber auch an Lehramtsstudien, die keine Verbeamtung ermöglichen.

\footnotetext{
${ }^{26}$ Als Beispiel eine Anzeige des Goethe-Instituts Mexiko, in der Diplomado und Diplomstudium verwechselt werden: https://www.goethe.de/ins/mx/de/kul/sup/fsf/dpl.html.

${ }^{27}$ Vgl. Bundesministerium für Justiz und für Verbraucherschutz (Stand: 2019): Hochschulrahmengesetz in der Fassung der Bekanntmachung vom 19. Januar 1999 (BGBl. I S. 18), das zuletzt durch Artikel 1 des Gesetzes vom 15. November 2019 (BGBl. I S. 1622) geändert worden ist, §§ 42-57.
} 
GRUHN, D. H. - Deutsch $\leftrightarrow$ Spanisch - Übersetzuns

\section{Ausblick: Interaktive Datenbanken?}

Angesichts der immer komplexer werdenden und in ständigem Wandel begriffenen Bildungslandschaften, werden länderspezifische ein- und zweisprachige Fachglossare dringender denn je benötigt. „Infolge der immer intensiveren internationalen Zusammenarbeit nehmen Menge und Umfang der zu übersetzenden Texte ständig zu. [...] Mit dieser Entwicklung können die fachsprachlichen Wörterbücher weder quantitativ noch qualitativ Schritt halten“, konstatierte Arntz schon 1999 (77). Dass diese Aufgabe auch heute noch längst nicht zufriedenstellend gelöst ist, wurde in vorigen Kapiteln dieser Arbeit bereits angedeutet. Zwar existieren einige (ein- oder mehrsprachige) Glossare, diese weisen aber noch erhebliche Mängel auf, viele sind wenig umfassend oder seit Jahren nicht aktualisiert worden, teilweise auch mit Fehlern behaftet. Erschwert wird die terminologische Arbeit dadurch, dass für viele Begriffe keine offiziellen Definitionen vorliegen. Ein solches Fachglossar immer auf dem neuesten Stand zu halten, ist mit hohem (Personal-, Zeit-, Kosten-) Aufwand verbunden. Anzudenken wären digitale Datenbanken in interaktiven Formaten, bei denen eine Vielzahl an Nutzern aus verschiedenen Ländern mitwirken können, indem sie Einträge anbringen, kommentieren oder aktualisieren, sowie Kontextbeispiele und Dokumente als Belege beifügen.

Nicht zuletzt beunruhigt die gegenwärtige Tendenz, bei deutschen Amtsstellen Übersetzungen vom Spanischen ins Englische zu verlangen bzw. wahlweise anzunehmen. Für viele Begriffe des deutschen Bildungswesens gibt es aber in der englischen Sprache ebensowenig wie im Spanischen Äquivalente bzw. äquivalente Benennungen, womit Ungenauigkeiten und Missverständnisse vorprogrammiert sind.

\section{Literaturverzeichnis}

ARBEITSGRUPPE Bildungsbericht am Max-Planck-Insitut für Bildungsforschung. Das Bildungswesen in der Bundesrepublik Deutschland. Reinbek bei Hamburg: Rowohlt Taschenbuch Verlag, 1994.

ARNTZ, Reiner. Terminologie der Terminologie. In: HoRnBY, Mary Snell; HÖNIG, Hans G.; KußMaul, Paul; SchmitT, Peter A. (Hg.). Handbuch Translation. Tübingen: Stauffenburg Verlag, 1999, 77-82.

ARNTZ, Reiner; PICHT, Heribert; SCHMITZ, Klaus-Dirk. Einführung in die Terminologiearbeit. Hildesheim: Olms-Verlag, 2014. 


$$
\text { GRUHN, D. H. - Deutsch } \leftrightarrow \text { Spanisch - Übersetzung }
$$

BDÜ, Landesverband Bayern. Allgemeine Leitlinie für die Anfertigung von Urkundenübersetzungen in Bayern. 2012. Verfügbar unter: https://by.bdue.de/fileadmin/verbaende/by/Dateien/_processed_/f/3/csm_BDUE_Bayern _Leitlinie_Urkundenuebersetzen_3565ef0db2.jpg (15/01/2021).

BERMAN, Antoine. La traduction et la lettre ou l'auberge du lointain. In: BERMAN, Antoine. Les tours de Babel. Essais sur la traduction. Mauzevin: Trans-Europe-Repress, 35-150, 1985.

DAAD. Diccionario / Wörterbuch. Terminología de la educación superior / Begriffe aus Wissenschaft und Hochschule. 2. Aufl. Bonn: Deutscher Akademischer Austauschdienst, 1998.

DUdEN. Recht A-Z. Fachlexikum für Studium, Ausbildung und Beruf. Berlin: Dudenverlag, 2015.

FLECK, Klaus E.W. Urkundenübersetzung. In: HORNBY, Mary Snell; HÖNIG, Hans G.; KUßMAUL, Paul; SCHMITT, Peter A. (Hg.). Handbuch Translation. 2. Aufl. Tübingen: Stauffenburg Verlag, 230-234, 1999.

FREIE und Hansestadt Hamburg. Behörde für Inneres und Sport. Merkblatt für die Anfertigung von beglaubigten Übersetzungen. 2010. Verfügbar unter: https://www.hamburg.de/contentblob/2111860/0239e6468b043a0edfcaf72f026a25ea/da ta/merkblatt-uebersetzungen.pdf (11/04/2021).

FUNDACIÓN Italia Morayta. Estudio de encuesta sobre la traducción y la interpretación en México 2017. Mexiko-Stadt: Fundación Italia Morayta, 2017.

HENNEQUiN, Jean. La traducción legale. Lenguas en Contexto, n. 5, 75-80, 2008.

House, Juliane. Offene und verdeckte Übersetzung: Zwei Arten, in einer anderen Sprache 'das Gleiche' zu sagen. Zeitschrift für Literaturwissenschaft und Linguistik, v. 35, n. 3, 76101, 2005.

INSTITUTO Nacional de Estadística, Geografía e Informática (INEGI). Clasificación Mexicana de Ocupaciones (CMO) - Histórica. Volumen I. Mexiko, o.J.

KADE, Otto. Zufall und Gesetzmäßigkeit in der Übersetzung. Beihefte zur Zeitschrift Fremdsprachen I. Leipzig: VEB-Verlag, 1968.

KOLLER, Werner. Einführung in die Übersetzungswissenschaft. Stuttgart: UTB, 2011.

KULTUSMINISTERKONFERENZ - Zentralstelle für ausländisches Bildungswesen. anabin. Das Inforportal zu ausländischen Bildungsabschlüssen, 2010-2021. Verfügbar unter: https://anabin.kmk.org/anabin.html (10/04/2021).

NORD, Christiane. Das Verhältnis des Zieltexts zum Ausgangstext. In: SNELL-HoRnBY, Mary; HÖNIG, Hans G.; KußMAul, Paul; SchmitT, Peter A. (Hg.). Handbuch Tanslation. Tübingen: Stauffenburg, 141-144, 1999.

o.A. Documentos. La educación Normal en México. Elementos para su análisis. Perfiles Educativos, v. 40, n. 160, 192-208, 2018. Verfügbar unter: http://www.scielo.org.mx/scielo.php?script=sci_issuetoc $\&$ pid=0185$269820180002 \& \operatorname{lng}=$ es\&nrm=iso $(10 / 04 / 2021)$.

ReICHMANN, Tinka. Die Textsorte „Notenspiegel“ im Hochschulkontext. Didkaktische Anregungen für den Übersetzungsunterricht Deutsch / Portugiesich. In: FANDRYCH, Christian; Galván ToRres, Adriana R.; HeIDERMAnN, Werner; Plesß, Ulrike; TSCHIRNER, Erwin (Hg.). Text, Diskurs und Translation im Wandel. Transformationen in der lateinamerikanischen Germanistik. Tübingen: Stauffenburg Verlag, 2013, 101-110.

REIß, Katharina; VERMEER, Hans J. Grundlegung einer allgemeinen Translationstheorie. Berlin: De Gruyter, 1984.

SCHLEIERMACHER, Friedrich. „Ueber die verschiedenen Methoden des Uebersezens“. In: STÖRIG, Hans Joachim (Hg.). Das Problem des Übersetzens. Stuttgart: H. Goverts, 1963, 38-70 [1813].

SEP (Secretaría de Educación Pública). Comunicado 269. 2016. Verfügbar unter: https://www.gob.mx/sep/prensa/comunicado-269-senala-nuno-mayer-importancia-de-

Pandaemonium, São Paulo, v. 25, n. 45, jan.-abr. 2022, p. 83-109 


$$
\text { GRUHN, D. H. - Deutsch } \leftrightarrow \text { Spanisch - Übersetzuns }
$$

abrir-abanico-de-competencia-entre-escuelas-normales-y-universidades?idiom=es (13/04/2021).

SEP (Secretaría de Educación Pública). Glosario de términos educativos. 2008. Verfügbar unter: http://cumplimientopef.sep.gob.mx/2010/Glosario\%202008\%2024-jun-08.pdf (21/01/2021).

SEP (Secretaría de Educación Pública). Glosario educación superior. o.J. Verfügbar unter: http://dsia.uv.mx/cuestionario911/material_apoyo/glosario\%20911.pdf (21/01/2021).

SIEVER, Holger. Übersetzungswissenschaft. Eine Einführung. Tübingen: Narr, 2015.

STOLZE, Radegundis. Fachübersetzen - Ein Lehrbuch für Theorie und Praxis. 3. Aufl. Berlin: Frank \& Timme, 2013.

STOLZE, Radegundis. Übersetzungstheorien. Eine Einführung. Tübingen: Narr, 2011.

THIRY, Bernard. Diccionario jurídico. Terminología de la responsabilidad civil (español-francés (francés-español). Granada: Editorial Conares, 2005.

THIRY, Bernard. Propiedad y contagio en el comparatismo y traducción jurídica. In: III Congreso «El Español, Lengua de Traducción» Contacto y contagio, 2006, Puebla (México). Actas. Bruselas: Asociación ESLETRA, 2008, 525-543.

UNIVERSIDAD Nacional Autónoma de México (UNAM). Gran Diccionario Náhuatl. 2012. Verfügbar unter: http://www.gdn.unam.mx (13/04/2021).

VENUTI, Lawrence. The Translator's Invisibility. Routledge, New York, 1995.

Recebido em 16 de fevereiro de 2021 Aceito em 9 de junho de 2021 